\title{
INTRODUCTION TO SCANNING TUNNELING MICROSCOPY
}

\author{
SECOND EDITION \\ C. JULIAN CHEN \\ Department of Applied Physics and Applied Mathematics, \\ Columbia University, New York
}




\section{Contents}

Preface to the Second Edition

xxiii

Preface to the First Edition xxvii

Gallery

xxxiii

Chapter 1: Overview 1

1.1 The scanning tunneling microscope . . . . . . . . . . . 1

1.2 The concept of tunneling . . . . . . . . . . . . . 3

1.2.1 Transmission coefficient . . . . . . . . . . 3

1.2.2 Semiclassical approximation . . . . . . . . . 6

1.2.3 The Landauer theory . . . . . . . . . . . . . . . . 6

1.2.4 Tunneling conductance . . . . . . . . . . . . 10

1.3 Probing electronic structure at atomic scale . . . . . . . . 12

1.3.1 Experimental observations . . . . . . . . . . . 15

1.3.2 Origin of atomic resolution in STM . . . . . . . . 18

1.4 The atomic force microscope . . . . . . . . . . . 21

1.4.1 Atomic-scale imaging by AFM . . . . . . . . . . 21

1.4.2 Role of covalent bonding in AFM imaging . . . . . . . 24

1.5 Illustrative applications $\ldots \ldots \ldots \ldots \ldots \ldots$

1.5.1 Catalysis research . . . . . . . . . . . . . 25

1.5.2 Atomic-scale imaging at the liquid-solid interface . . . 29

1.5.3 Atom manipulation . . . . . . . . . . . . 33

1.5.4 Imaging and manipulating DNA using AFM $\ldots \ldots 35$

$\begin{array}{lll}\text { Part I Principles } & 41\end{array}$

Chapter 2: Tunneling Phenomenon 45

2.1 The metal-insulator-metal tunneling junction . . . . . . . 46

2.2 The Bardeen theory of tunneling . . . . . . . . . 48

2.2 .1 One-dimensional case . . . . . . . . . . . 48

2.2.2 Tunneling spectroscopy . . . . . . . . . . . . 52

2.2.3 Energy dependence of tunneling matrix elements . . . 53

2.2.4 Asymmetry in tunneling spectrum ... . . . . . 5 54

2.2.5 Three-dimensional case . . . . . . . . . . 57

2.2.6 Error estimation . . . . . . . . . . . . . 59

2.2.7 Wavefunction correction . . . . . . . . . . 60

2.2 .8 The transfer-Hamiltonian formalism . . . . . . . 61

2.2 .9 The tunneling matrix $\ldots \ldots \ldots \ldots$ 
2.2.10 Relation to the Landauer theory . . . . . . . . 64

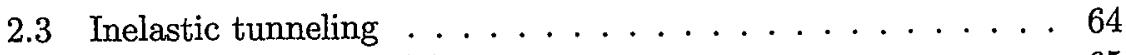

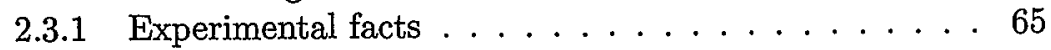

2.3.2 Frequency condition ........... 66

2.3.3 Effect of finite temperature ......... 67

2.4 Spin-polarized tunneling . . . . . . . . . . . . . . . 69

2.4.1 General formalism ............... 70

2.4.2 The spin-valve effect .............. 72

2.4.3 Experimental observations . . . . . . . . 76

$\begin{array}{ll}\text { Chapter 3: Tunneling Matrix Elements } & 77\end{array}$

3.1 Introduction . . . . . . . . . . . . . 77

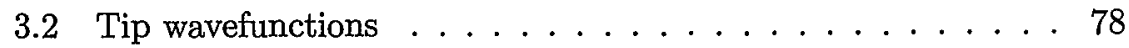

3.2.1 General form . . . . . . . . . . . . 78

3.2.2 Tip wavefunctions as Green's functions . . . . . . 81

3.3 The derivative rule: individual cases . . . . . . . . . . 82

3.3.1 s-wave tip state ............... 82

3.3.2 $p$-wave tip states ............... 83

3.3.3 $d$-wave tip states ................ 84

3.3.4 Complex tip states .............. 84

3.4 The derivative rule: general case ............ 85

3.5 An intuitive interpretation . . . . . . . . . . . . 91

Chapter 4: Atomic Forces $\quad 93$

4.1 Van $\operatorname{der}$ Waals force ... . . . . . . . . . . . . 93

4.1.1 The van der Waals equation of state . . . . . . . . 93

4.1.2 The origin of van der Waals force . . . . . . . . . . . . 94

4.1.3 Van der Waals force between a tip and a sample . . . 96

4.2 Hard-core repulsion . . . . . . . . . . . . . . . . . 98

4.3 The ionic bond . . . . . . . . . . . . . . . 98

4.4 The covalent bond: The concept . . . . . . . . . . . . 100

4.4.1 Heisenberg's model of resonance . . . . . . . . . . . 101

4.4.2 The hydrogen molecule-ion . . . . . . . . . . . . 104

4.4.3 Three regimes of interaction . . . . . . . . . . . 105

4.4.4 Van der Waals force ... . . . . . . . . . . 106

4.4.5 Resonance energy as tunneling matrix element . . . . 107

4.4.6 Evaluation of the modified Bardeen integral . . . . . . 111

4.4.7 Repulsive force . . . . . . . . . . . . . . 114

4.5 The covalent bond: Many-electron atoms . . . . . . . 115 
4.5.1 The homonuclear diatomic molecules . . . . . . . . 115

4.5.2 The perturbation approach . . . . . . . . . 115

4.5.3 Evaluation of the Bardeen Integral . . . . . . . . . . 118

4.5.4 Comparison with experimental data . . . . . . . . 119

Chapter 5: Atomic Forces and Tunneling

5.1 The principle of equivalence $\ldots \ldots \ldots \ldots \ldots \ldots$

5.2 General theory . . . . . . . . . . . . . . . . 126

5.2 .1 The double-well problem . . . . . . . . . . . . . 126

5.2.2 Canonical transformation of the transfer Hamiltonian 128

5.2.3 Diagonizing the tunneling matrix . . . . . . . . 130

5.3 Case of a metal tip and a metal sample . . . . . . . . 131

5.3.1 Van der Waals force . . . . . . . . . . . . . . . 132

5.3.2 Resonance energy between two metal electrodes . . . . 132

5.3.3 A measurable consequence . . . . . . . . . . . . . 135

5.3 .4 Repulsive force . . . . . . . . . . . . . 136

5.4 Experimental verifications . . . . . . . . . . . 136

5.4 .1 An early experiment . . . . . . . . . . . . . 136

5.4.2 Experiments with frequency-modulation AFM . . . 138

5.4.3 Experiments with static AFM . . . . . . . . . . . 140

5.4.4 Non-contact atomic force spectroscopy . . . . . . . . 143

5.5 Threshold resistance in atom manipulation . . . . . . 145

Chapter 6: Nanometer-Scale Imaging 149

6.1 Types of STM and AFM images . . . . . . . . . 149

6.2 The Tersoff-Hamann model . . . . . . . . . . . . 151

6.2 .1 The concept . . . . . . . . . . . . . . 151

6.2 .2 The original derivation . . . . . . . . . . . 152

6.2.3 Profiles of surface reconstructions . . . . . . . . 155

6.2.4 Extension to finite bias voltages . . . . . . . 158

6.2.5 Surface states: the concept . . . . . . . . . . 160

6.2 .6 Surface states: STM observations . . . . . . . . . 162

6.2.7 Heterogeneous surfaces . . . . . . . . . . . . 166

6.3 Limitations of the Tersoff-Hamann model . . . . . . . . 166

Chapter 7: Atomic-Scale Imaging $\quad 169$

7.1 Experimental facts . . . . . . . . . . . . . . 170

7.1.1 Universality of atomic resolution . . . . . . . . 170

7.1 .2 Corrugation inversion . . . . . . . . . . . 170

7.1 .3 Tip-state dependence $\ldots \ldots \ldots$. . . . . . 171 
7.1.4 Distance dependence of corrugation . . . . . . 173

7.2 Intuitive explanations . . . . . . . . . . . . . . 174

7.2.1 Sharpness of tip states . . . . . . . . . . . 174

7.2 .2 Phase effect . . . . . . . . . . . . . 175

7.2.3 Arguments based on the reciprocity principle . . . 177

7.3 Analytic treatments . . . . . . . . . . . . . . 178

7.3.1 A one-dimensional case .............. 178

7.3.2 Surfaces with hexagonal symmetry . . . . . . 182

7.3.3 Corrugation inversion .............. 186

7.3.4 Profiles of atomic states as seen by STM . . . . . . 190

7.3.5 Independent-orbital approximation . . . . . . . . 194

7.4 First-principles studies: tip electronic states . . . . . . . . . . 198

7.4.1 W clusters as STM tip models . . . . . . . . . . 198

7.4.2 Density-functional study of a W-Cu STM junction . . 199

7.4.3 Transition-metal pyramidal tips . . . . . . . . . 199

7.4.4 Transition-metal atoms adsorbed on W slabs . . . . 200

7.5 First-principles studies: the images . . . . . . . . . . . . 202

7.5.1 Transition-metal surfaces ............. 202

7.5.2 Atomic corrugation and surface waves . . . . . . . . 204

7.5.3 Atom-resolved AFM images ............ . 205

7.6 Spin-polarized STM . . . . . . . . . . . . . 209

7.7 Chemical identification of surface atoms . . . . . . . . . 212

7.8 The principle of reciprocity . . . . . . . . . . . . 214

Chapter 8: Nanomechanical Effects 219

8.1 Mechanical stability of the tip-sample junction . . . . . . 220

8.1.1 Experimental observations . . . . . . . . . . . 220

8.1.2 Condition of mechanical stability .......... 223

8.1.3 Relaxation and the apparent $G \sim z$ relation . . . . . 229

8.2 Mechanical effects on observed corrugations . . . . . . . 231

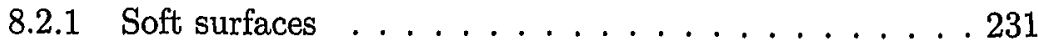

8.2 .2 Hard surfaces ................. . . 233

8.2.3 First-principles simulations ........... 236

8.2.4 Advanced topics ................. 237

8.2.5 The Pethica mechanism ............. 238

8.3 Force in tunneling-barrier measurements . . . . . . . . 238 


\section{Part II Instrumentation}

Chapter 9: Piezoelectric Scanner

9.1 Piezoelectricity . . . . . . . . . . . . . . . . . . . 245

9.1.1 Piezoelectric effect . . . . . . . . . . . . . . 245

9.1 .2 Inverse piezoelectric effect . . . . . . . . . . . . 246

9.2 Piezoelectric materials in STM and AFM . . . . . . . . 249

9.2 .1 Quartz . . . . . . . . . . . . . . . . . . 249

9.2.2 Lead zirconate titanate ceramics . . . . . . . . . 250

9.3 Piezoelectric devices in STM and AFM . . . . . . . 254

9.3.1 Tripod scanner . . . . . . . . . . . . . . 254

9.3.2 Bimorph . . . . . . . . . . . . . . 255

9.4 The tube scanner . . . . . . . . . . . . . 257

9.4.1 Deflection . . . . . . . . . . . . . . 258

9.4 .2 In situ testing and calibration . . . . . . . . . . 260

9.4.3 Resonant frequencies . . . . . . . . . . . . . . . 263

9.4.4 Tilt compensation: the s-scanner . . . . . . . . . . 264

9.4.5 Repolarizing a depolarized tube piezo . . . . . . . . 265

9.5 The shear piezo . . . . . . . . . . . . . . . 265

Chapter 10: Vibration Isolation 269

10.1 Basic concepts . . . . . . . . . . . . . . 269

10.2 Environmental vibration . . . . . . . . . . . . 273

10.2.1 Measurement method . . . . . . . . . . . . 274

10.2.2 Vibration isolation of the foundation . . . . . . 275

10.3 Vibrational immunity of STM . . . . . . . . . . . 277

10.4 Suspension-spring systems . . . . . . . . . . . . . 278

10.4.1 Analysis of two-stage systems . . . . . . . . . . 278

10.4.2 Choice of springs . . . . . . . . . . . . . . . 280

10.4.3 Eddy-current damper . . . . . . . . . . . . 281

10.5 Pneumatic systems . . . . . . . . . . . . . . 282

Chapter 11: Electronics and Control 283

11.1 Current amplifier . . . . . . . . . . . . . . . . . 283

11.1.1 Johnson noise and shot noise . . . . . . . . . 284

11.1 .2 Frequency response . . . . . . . . . . . . . . . 286

11.1.3 Microphone effect . . . . . . . . . . . . . . . . . . 287

11.1.4 Logarithmic amplifier . . . . . . . . . . . . . 288

11.2 Feedback circuit . . . . . . . . . . . . . . . . . 289 
11.2.1 Steady-state response . . . . . . . . . . . . . 290

11.2.2 Transient response . . . . . . . . . . . . . . . . . 292

11.3 Computer interface . . . . . . . . . . . . . . . . . . 297

11.3.1 Automatic approaching . . . . . . . . . . . 298

Chapter 12: Mechanical design 299

12.1 The louse . . . . . . . . . . . . . . . . . . 299

12.2 The pocket-size STM . . . . . . . . . . . . . 300

12.3 The single-tube STM . . . . . . . . . . . . . . . . . 301

12.4 The Besocke-type STM: the beetle . . . . . . . . . . . 302

12.5 The walker $\ldots \ldots \ldots \ldots \ldots \ldots \ldots \ldots$

12.6 The kangaroo . . . . . . . . . . . . . 306

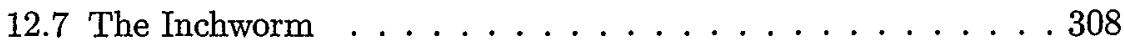

12.8 The match . . . . . . . . . . . . . . . . 309

Chapter 13: Tip Treatment 313

13.1 Introduction . . . . . . . . . . . . . . . . . . . . . .

13.2 Electrochemical tip etching . . . . . . . . . . . . . . 314

13.3 Ex situ tip treatments . . . . . . . . . . . . . . . . 317

13.3.1 Annealing . . . . . . . . . . . . . . . . . 317

13.3.2 Field evaporation and controlled deposition . . . . . 318

13.3.3 Annealing with a field . . . . . . . . . . . . . . 319

13.3.4 Atomic metallic ion emission . . . . . . . . . . . 320

13.3.5 Field-assisted reaction with nitrogen . . . . . . . . 322

13.4 In situ tip treatments . . . . . . . . . . . . . . . . . . . 324

13.4.1 High-field treatment . . . . . . . . . . . . . . . 324

13.4 .2 Controlled collision . . . . . . . . . . . . . . . 325

13.5 Tip treatment for spin-polarized STM . . . . . . . . . . 326

13.5.1 Coating the tip with ferromagnetic materials . . . . 326

13.5.2 Coating the tip with antiferromagnetic materials . . . 327

13.5.3 Controlled collision with magnetic surfaces . . . . . 327

13.6 Tip preparation for electrochemistry STM . . . . . . . . . 328

Chapter 14: Scanning Tunneling Spectroscopy 331

14.1 Electronics for scanning tunneling spectroscopy . . . . . . . 331

14.2 Nature of the observed tunneling spectra . . . . . . . . . 332

14.3 Tip treatment for spectroscopy studies . . . . . . . . . . 334 
14.3.1 Annealing . . . . . . . . . . . . . . . . . . 334

14.3.2 Controlled collision with a metal surface . . . . . . 336

14.4 The Feenstra parameter . . . . . . . . . . . . . 337

14.5 Determination of the tip DOS . . . . . . . . . . 338

14.5.1 Ex situ methods . . . . . . . . . . . . . 338

14.5.2 In situ methods . . . . . . . . . . . . . . . 340

14.6 Inelastic scanning tunneling spectroscopy . . . . . . . . . 344

14.6.1 Instrumentation . . . . . . . . . . . . . . 344

14.6.2 Effect of finite modulation voltage . . . . . . . . . . 345

14.6.3 Experimental observations . . . . . . . . . . . . . 347

Chapter 15: Atomic Force Microscopy 349

15.1 Static mode and dynamic mode . . . . . . . . . . 350

15.2 The cantilever . . . . . . . . . . . . . . . 351

15.2.1 Basic requirements . . . . . . . . . . . . . 351

15.2.2 Fabrication . . . . . . . . . . . . . . . 352

15.3 Static force detection . . . . . . . . . . . . . . 354

15.3.1 Optical beam deflection . . . . . . . . . . . 354

15.3.2 Optical interferometry . . . . . . . . . . . . 356

15.4 Tapping-mode AFM . . . . . . . . . . . . . . . 357

15.4.1 Acoustic actuation in liquids . . . . . . . . . 358

15.4.2 Magnetic actuation in liquids . . . . . . . . . . . 359

15.5 Non-contact AFM . . . . . . . . . . . . . . . 361

15.5.1 Case of small amplitude . . . . . . . . . . . . 361

15.5.2 Case of finite amplitude . . . . . . . . . . . . . 364

15.5.3 Response function for frequency shift . . . . . . . 365

15.5.4 Second harmonics . . . . . . . . . . . . 366

15.5.5 Average tunneling current . . . . . . . . . . 368

15.5.6 Implementation . . . . . . . . . . . . . . . . . . . . . . . . .

Appendix A: Green's Functions $\quad 371$

Appendix B: Real Spherical Harmonics 373

Appendix C: Spherical Modified Bessel Functions $\quad 377$

Appendix D: Plane Groups and Invariant Functions 381

D.1 A brief summary of plane groups . . . . . . . . . . 382

D.2 Invariant functions $\ldots \ldots \ldots \ldots \ldots \ldots \ldots$ 
Appendix E: Elementary Elasticity Theory

E.1 Stress and strain . . . . . . . . . . . . . . . . . 389

E.2 Small deflection of beams . . . . . . . . . . . . 391

E.3 Vibration of beams . . . . . . . . . . . . . . . . . 394

E.4 Torsion . . . . . . . . . . . . . . . . . . 395

E.5 Helical springs . . . . . . . . . . . . . . . . . . 397

E.6 Contact stress: The Hertz formulas . . . . . . . . . . . . 398 